\title{
Theory of Four-dimensional Fractional Quantum Hall States
}

\author{
Chyh-Hong Chern* \\ ERATO-SSS, Department of Applied Physics, University of Tokyo, Bunkyo-ku, Tokyo 113-8656, Japan
}

\begin{abstract}
We propose a pseudo-potential Hamiltonian for the Zhang-Hu's generalized fractional quantum Hall states to be the exact and unique ground states. Analogously to Laughlin's quasi-hole (quasiparticle), the excitations in the generalized fractional quantum Hall states are extended objects. They are vortex-like excitations with fractional charges $+(-) 1 / m^{3}$ in the total configuration space $\mathrm{CP}^{3}$. The density correlation function of the Zhang-Hu states indicates that they are incompressible liquid.

PACS numbers:
\end{abstract}

Recently, the four-dimensional generalization of the quantum Hall effect (4DQHE) proposed by Zhang and $\mathrm{Hu}(\mathrm{ZH})$ has drawn considerable attentions in both condensed matter physics and high energy physics communities 1]. The projected generalized Hall conductivity is considered to be the root of the spin Hall effect in the $p$-type semiconductor 2]. When applying the electric field to the $p$-type semiconductor, the dissipationless spin current flows perpendicular to the direction of the electric field. The purely electrical manipulation of the spin current is one of the ultimate goals for the applications in the information technology. On the other hand, 4DQHE intrigues people by its topological property [3] and the deep relation with the division algebra 4]. Furthermore, numerous works have been done in the language of the modern string theory [5, 6], part of which are motivated by the massless edge excitations which carry integer spins with helicity [7]. The underlying noncommutative feature of $\mathrm{ZH}$ theory also fosters intensive studies in the non-commutative geometry in the higher dimensional compact spaces [8].

Besides the generalized integer quantum Hall effect, $\mathrm{ZH}$ also proposed a wave function with fractional filling, which they referred to the generalized fractional quantum Hall states (FQHS). In two dimensions, the integer QHE can be well understood by the one-body quantum mechanics but in the fractional case it is the two-body repulsive Coulombic interaction which plays the dominant role for the new states of matter possessing the excitations with fractional charges and fractional statistics 9 ]. Similarly, the ZH fractional quantum Hall states are believed to have many interesting properties, namely being disordered in higher dimensions and the excitations with fractional charges. Besides, the edge modes of the FQHS are still far from clear. Therefore, it is urgent to understand the many-body interaction in the ZH FQHS. In this paper, we will try to answer this question. Namely, we propose the two-body interaction Hamiltonian for the FQHS to be the non-degenerate ground state. Furthermore, the correspondent Laughlin's quasi-particle (quasihole) in the 4D FQHS can be shown as a four-dimensional extended object which can be considered as the vortex in the total configuration space $\mathrm{CP}^{3}$. In addition, we will show that the vortex-like excitations carry the fractional charge $+(-) 1 / m^{3}$.

Let us start with a brief review of ZH's construction. The non-consecutive jump from two dimensions to four dimensions results from the underlying algebraic structures. In two dimensions, the two-dimensional complex spinor coordinate $\phi^{\alpha}$ used to construct the coherent state on the two-sphere can be introduced by the first Hopf map, that is $X_{i} / R=\bar{\phi}^{\alpha}\left(\sigma_{i}\right)_{\alpha \beta} \phi^{\beta}$, where $X_{i}$ are the coordinates on the two-sphere, $R$ is the radius, and $\sigma_{i}$ are the Pauli matrices. ZH generalized it by considering the second Hopf map, which is $X_{a} / R=\bar{\psi}^{\alpha}\left(\Gamma_{a}\right)_{\alpha \beta} \psi^{\beta}$, where $X_{a}$ are the coordinates on the four-sphere, $R$ is the radius, and $\Gamma_{a}$ are the $\mathrm{SO}(5)$ Gamma matrices given by

$$
\Gamma^{i}=\left(\begin{array}{cc}
0 & i \sigma_{i} \\
-i \sigma_{i} & 0
\end{array}\right), \Gamma^{4}=\left(\begin{array}{ll}
0 & 1 \\
1 & 0
\end{array}\right), \Gamma^{5}=\left(\begin{array}{cc}
1 & 0 \\
0 & -1
\end{array}\right)
$$

where $i$ is from 1 to 3 . An explicit solution $\psi^{\alpha}$ of the second Hopf map can be obtained as

$$
\begin{aligned}
& \left(\begin{array}{l}
\psi^{1} \\
\psi^{2}
\end{array}\right)=\sqrt{\frac{R+X_{5}}{2 R}\left(\begin{array}{c}
\phi^{1} \\
\phi^{2}
\end{array}\right),} \\
& \left(\begin{array}{l}
\psi^{3} \\
\psi^{4}
\end{array}\right)=\sqrt{\frac{1}{2 R\left(R+X_{5}\right)}}\left(X_{4}-i X_{i} \sigma_{i}\right)\left(\begin{array}{c}
\phi^{1} \\
\phi^{2}
\end{array}\right)
\end{aligned}
$$

where implicit summation is assumed and $\left(\phi^{1}, \phi^{2}\right)$ is an arbitrary complex spinor with $\bar{\phi}^{i} \phi_{i}=1$. One can define the $\mathrm{SU}(2)$ gauge field $a_{a}$ from Eq.(2) as $\bar{\psi}^{\alpha} d \psi_{\alpha}=\bar{\phi}^{\alpha}\left(a_{a} d x_{a}\right)_{\alpha \beta} \phi^{\beta}$, where the dimensionless coordinate $x_{a}=X_{a} / R$ is used. The field strength $f_{a b}$ can be defined by $\left[D_{a}, D_{b}\right]$, where $D_{a}$ is the covariant derivative. Then, the Hamiltonian can be written as $H=\frac{\hbar^{2}}{2 M R^{2}} \sum_{a<b} \Lambda_{a b}^{2}$, where $\Lambda_{a b}=-i\left(x_{a} D_{b}-x_{b} D_{a}\right)$. Introducing $L_{a b}=\Lambda_{a b}-i f_{a b}$, the Hamiltonian can be expressed as $H=\frac{\hbar^{2}}{2 M R^{2}}\left(\sum_{a<b} L_{a b}^{2}-2 I_{i}^{2}\right)$, where $I$ denotes the representation of the $\mathrm{SU}(2)$ gauge group. $L_{a b}$ can be shown to satisfy the $\mathrm{SO}(5)$ algebra. Therefore, the quantum Hall states can be classified into the $\mathrm{SO}(5)$ representations labelled by two integers $(p, q)$. Given $I, p$ can be related by $p=2 I+q$. The spectrum of the generalized quantum Hall effect is $E(2 I+q, q)=\frac{\hbar^{2}}{2 M R^{2}}(C(2 I+$ $q, q)-2 I(I+1))$, where $C(p, q)=p^{2} / 2+q^{2} / 2+2 p+q$ 
is one of the Casimir operator of the $\mathrm{SO}(5)$ group, and $q$ is the Landau level index. 1].

Larger symmtry in the lowest Landau level The lowest Landau level (lll) is described by the $\mathrm{SO}(5)(p, 0)$ representation, namely $q=0$. The degeneracy is given by $d(p, 0)=\frac{1}{6}(p+1)(p+2)(p+3)$. The single-particle wave function in the lll can be described only by the half of the coordinates $\psi^{\alpha}$

$$
\sqrt{\frac{p !}{m_{1} ! m_{2} ! m_{3} ! m_{4} !}}\left(\psi^{1}\right)^{m_{1}}\left(\psi^{2}\right)^{m_{2}}\left(\psi^{3}\right)^{m_{3}}\left(\psi^{4}\right)^{m_{4}}
$$

where $m_{i}$ are non-negative integers with $\sum_{i=1}^{4} m_{i}=p$. To have finite energy in the lll, $p$ has to be proportional to $R^{2}$. The magnetic length $l_{0}$ can be defined as $l_{0}=\lim _{R \rightarrow \infty} R / \sqrt{p}$. Furthermore, in the large- $p$ limit, the degeneracy in the 111 is proportional to $p^{3}$, which is proportional to $R^{6}$. It is because the $\mathrm{SU}(2)$ gauge group introduces additional internal degrees of freedom which is $S^{2}$. The total configuration space of 111 counts from the internal degrees of freedom $S^{2}$ and the orbital one $S^{4}$. Locally, $S^{4} \times S^{2}$ is isomorphic to $\mathrm{CP}^{3}$ which is the six-dimensional complex projective space and the coordinates are $\left(X_{1}, X_{2}, X_{3}, X_{4}, X_{5}\right)$ with $\sum_{i=1}^{5} X_{i}=R^{2}$ for the orbital and $\left(n_{1}, n_{2}, n_{3}\right)$ with $\sum_{i=1}^{3} n_{i}=r^{2}$ for the internal degrees of freedom. From $\psi^{\alpha}, n_{i}$ is given by $n_{i} / r=\bar{\phi}^{\alpha}\left(\sigma_{i}\right)_{\alpha \beta} \phi^{\beta} . \psi^{\alpha}$ actually describes a spinor on $\mathrm{CP}^{3}$. When the number of particles $N=d(p, 0)$, lll is fully filled. The many-body wavefunction $\Psi$ is the Slater determinant of Eq.(3) which is proportional to

$$
\Psi=\left|\begin{array}{cccc}
\left(\psi_{1}^{1}\right)^{p} & \left(\psi_{1}^{1}\right)^{p-1} \psi_{1}^{2} & \cdots & \left(\psi_{1}^{4}\right)^{p} \\
\left(\psi_{2}^{1}\right)^{p} & \left(\psi_{2}^{1}\right)^{p-1} \psi_{2}^{2} & \cdots & \left(\psi_{2}^{4}\right)^{p} \\
\cdot & \cdot & \cdot & \cdot \\
\cdot & \cdot & \cdot & \cdot \\
\left(\psi_{N}^{1}\right)^{p} & \left(\psi_{N}^{1}\right)^{p-1} \psi_{N}^{2} & \cdots & \left(\psi_{N}^{4}\right)^{p}
\end{array}\right|
$$

where the subscripts are the particle indices. For the fractional states, $\mathrm{ZH}$ considered $\Psi^{m}$. The singleparticle state becomes $(m p, 0)[\mathbb{1}]$. While keeping the number of particles $N=d(p, 0)$ fixed, the filling factor $d(p, 0) / d(m p, 0)$ approaches to $1 / m^{3}$ in the thermodynamic limit.

Besides SO(5) symmetry, the wavefunctions in the 111 have larger $\mathrm{SU}(4)$ symmetry, because $\mathrm{SU}(4)$ is the isometry group of $\mathrm{CP}^{3}$. The 11 wavefunction can also be described by the $\mathrm{SU}(4)$ representations which are denoted by three integers $\left(n_{1}, n_{2}, n_{3}\right)$. Additionally, the 111 wavefunction is described by the $\mathrm{SU}(4)(p, 0,0)$ states with the degeneracy $\frac{1}{6}(p+1)(p+2)(p+3)$ which is exactly the same as that of $\mathrm{SO}(5)(p, 0)$ states. Furthermore, the $\mathrm{SU}(4)$ coherent states are also given by Eq.(3). In this case, the single-particle state in the fractional case is described by the $\mathrm{SU}(4)(m p, 0,0)$ state. Because we only consider the fractional case in the 1ll, we do not care about the problem of the $\mathrm{SO}(5)$ covariance.
Consider the following Hamiltonian

$$
H=\sum_{i<j} \sum_{q=1, \text { odd }}^{q \leq m-2} \kappa_{q} P_{i j}^{(2 m p-2 q, q, 0)} .
$$

where $i$ and $j$ runs from 1 to $N$ and $\kappa_{q}$ are positive constants. $P_{i j}^{(2 m p-2 q, q, 0)}$ indicate the projection operator of the $(2 m p-2 q, q, 0)$ states which describe the two-fermion states when $q$ is odd. We will prove $\mathrm{ZH}$ fractional quantum Hall state $\Psi^{m}$ is the zero-energy state of Eq.(15).

The two-fermion state is the antisymmetric channel of the direct-product space of $(m p, 0,0) \otimes(m p, 0,0)$, which can be decomposed as the direct-sum of the SU(4) invariant subspaces:

$$
\left.(m p, 0,0) \otimes(m p, 0,0)\right|_{a}=\bigoplus_{q=1, \text { odd }}^{m p}(2 m p-2 q, q, 0)
$$

where $a$ denotes the antisymmetric cannels. For $m=1$, the subspace in Eq. (16) with the highest $\mathrm{SU}(3)$ weight is $(2 p-2,1,0)$, because a general SU(4) $(2 p-2 q, q, 0)$ state can be decomposed as the direct sum of the SU(3) states:

$$
\begin{aligned}
& (2 p-2 q, q, 0)=(2 p-q, 0)+(2 p-q-1,0) \\
& +(2 p-q-2,0)+. .+(q, 0) \\
& +(2 p-q-1,1)+(2 p-q-2,1)+. .+(q-1,1) \\
& +. . \\
& +(2 p-2 q, q)+(2 p-2 q-1, q)+. .+(0, q)
\end{aligned}
$$

In $\Psi^{m}$, the highest $\mathrm{SU}(3)$ weight is simply the $m^{\text {th }}$ power of $(2 p-1,0)$, namely $(2 m p-m, 0)$. The two-fermion state in $\Psi^{m}$ contains only up to $(2 m p-2 m, m, 0)$. Therefore, it is the zero-energy state of the Hamiltonian in the Eq.(15).

Argument for the uniqueness To show the nondegeneracy, let us focus on the odd $p$ for convenience. Particularly, we shall use $p=3$ and $m=3$ as an example to illustrate our method. In this case, Eq.(15) can be written as $H=\kappa_{1} \sum_{i<j} P_{i j}^{(16,1,0)}$. Let us call $\chi$ the ground state wave function, namely $H \chi=0$. Since we expect the ground state wave function to be uniform, with the completeness of the two-fermion states $\chi$ have to satisfy

$$
\left(P_{i j}^{(12,3,0)}+P_{i j}^{(8,5,0)}+P_{i j}^{(4,7,0)}+P_{i j}^{(0,9,0)}\right) \chi=\chi \forall(i j)
$$

To solve Eq. (8), we expand $\chi$ in the general form

$$
\sum_{\left\{\alpha_{j k}\right\}} C_{3}\left(\left\{\alpha_{j k}\right\}\right) \prod_{j=1}^{N} \prod_{k=1}^{9} \psi_{j}^{\alpha_{j k}},
$$

where $N=20$ is the number of particles, and $\alpha_{j k}$ runs from 1 to 4 , and the summation goes over all configurations of $\left\{\alpha_{j k}\right\}$, and finally $\prod_{k=1}^{9} \psi_{j}^{\alpha_{j k}}$ is the $j^{\text {th }}$ 
single-particle state denoted by $(9,0,0)$, which is proportional to Eq.(3). From Eq.(8), $C_{3}$ satisfies the following Schrödinger equation:

$$
\begin{aligned}
& C_{3}=\sum_{\left\{\beta_{i k}, \beta_{j k}\right\}}\left(A_{3}\left(\left\{\alpha_{i k}\right\},\left\{\alpha_{j k}\right\},\left\{\beta_{i k}\right\},\left\{\beta_{j k}\right\}\right)\right. \\
& +A_{5}\left(\left\{\alpha_{i k}\right\},\left\{\alpha_{j k}\right\},\left\{\beta_{i k}\right\},\left\{\beta_{j k}\right\}\right) \\
& +A_{7}\left(\left\{\alpha_{i k}\right\},\left\{\alpha_{j k}\right\},\left\{\beta_{i k}\right\},\left\{\beta_{j k}\right\}\right) \\
& \left.+A_{9}\left(\left\{\alpha_{i k}\right\},\left\{\alpha_{j k}\right\},\left\{\beta_{i k}\right\},\left\{\beta_{j k}\right\}\right)\right) C_{3} \quad \forall(i j)
\end{aligned}
$$

where the spinor indices of particles $i$ and $j$ are summed over, and $A_{q}$ are the tensors denoting the ClebschGordon (CG) coefficients for the projection operators $P_{i j}^{(18-2 q, q, 0)}$. In $(9,0,0)$ state, there are 9 symmetric indices. To make the CG coefficient from $(9,0,0)_{i} \times$ $(9,0,0)_{j}$ to $(18-2 q, q, 0)_{i j}$, we have to make $q$ antisymmetric pairs among the indices between particles $i$ and $j$ and totally symmetrize the rest of the indices. For example, for a particular $(i j) A_{q}$ can be given as the following:

$$
\begin{aligned}
& A_{3}\left(\left\{\alpha_{i k}\right\},\left\{\alpha_{j k}\right\},\left\{\beta_{i k}\right\},\left\{\beta_{j k}\right\}\right) \\
& =\frac{1}{N_{3}}\left(\delta_{\beta_{i 1}}^{\alpha_{i 1}} \delta_{\beta_{j 1}}^{\alpha_{j 1}}-\delta_{\beta_{j 1}}^{\alpha_{i 1}} \delta_{\beta_{i 1}}^{\alpha_{j 1}}\right)\left(\delta_{\beta_{i 4}}^{\alpha_{i 4}} \delta_{\beta_{j 4}}^{\alpha_{j 4}}-\delta_{\beta_{j 4}}^{\alpha_{i 4}} \delta_{\beta_{i 4}}^{\alpha_{j 4}}\right) \\
& \left(\delta_{\beta_{i 7}}^{\alpha_{i 7}} \delta_{\beta_{j 7}}^{\alpha_{j 7}}-\delta_{\beta_{j 7}}^{\alpha_{i 7}} \delta_{\beta_{i 7}}^{\alpha_{j 7}}\right)\left(\delta_{\beta_{i 2}}^{\alpha_{i 2}} \delta_{\beta_{i 3}}^{\alpha_{i 3}} \delta_{\beta_{i 5}}^{\alpha_{i 5}} \delta_{\beta_{i 6}}^{\alpha_{i 6}} \delta_{\beta_{i 8}}^{\alpha_{i 8}} \delta_{\beta_{i 9}}^{\alpha_{i 9}}\right. \\
& \delta_{\beta_{j 2}}^{\alpha_{j 2}} \delta_{\beta_{j 3}}^{\alpha_{j 3}} \delta_{\beta_{j 5}}^{\alpha_{j 5}} \delta_{\beta_{j 6}}^{\alpha_{j 6}} \delta_{\beta_{j 8}}^{\alpha_{j 8}} \delta_{\beta_{j 9}}^{\alpha_{j 9}}+\text { sym.) } \\
& A_{5}\left(\left\{\alpha_{i k}\right\},\left\{\alpha_{j k}\right\},\left\{\beta_{i k}\right\},\left\{\beta_{j k}\right\}\right) \\
& =\frac{1}{N_{5}}\left(\delta_{\beta_{i 1}}^{\alpha_{i 1}} \delta_{\beta_{j 1}}^{\alpha_{j 1}}-\delta_{\beta_{j 1}}^{\alpha_{i 1}} \delta_{\beta_{i 1}}^{\alpha_{j 1}}\right)\left(\delta_{\beta_{i 2}}^{\alpha_{i 2}} \delta_{\beta_{j 2}}^{\alpha_{j 2}}-\delta_{\beta_{j 2}}^{\alpha_{i 2}} \delta_{\beta_{i 2}}^{\alpha_{j 2}}\right) \\
& \left(\delta_{\beta_{i 3}}^{\alpha_{i 3}} \delta_{\beta_{j 3}}^{\alpha_{j 3}}-\delta_{\beta_{j 3}}^{\alpha_{i 3}} \delta_{\beta_{i 3}}^{\alpha_{j 3}}\right)\left(\delta_{\beta_{i 4}}^{\alpha_{i 4}} \delta_{\beta_{j 4}}^{\alpha_{j 4}}-\delta_{\beta_{j 4}}^{\alpha_{i 4}} \delta_{\beta_{i 4}}^{\alpha_{j 4}}\right) \\
& \left(\delta_{\beta_{i 7}}^{\alpha_{i 7}} \delta_{\beta_{j 7}}^{\alpha_{j 7}}-\delta_{\beta_{j 7}}^{\alpha_{i 7}} \delta_{\beta_{i 7}}^{\alpha_{j 7}}\right)\left(\delta_{\beta_{i 5}}^{\alpha_{i 5}} \delta_{\beta_{i 6}}^{\alpha_{i 6}} \delta_{\beta_{i 8}}^{\alpha_{i 8}} \delta_{\beta_{i 9}}^{\alpha_{i 9}} \delta_{\beta_{j 5}}^{\alpha_{j 5}} \delta_{\beta_{j 6}}^{\alpha_{j 6}}\right. \\
& \delta_{\beta_{j 8}}^{\alpha_{j 8}} \delta_{\beta_{j 9}}^{\alpha_{j 9}}+\text { sym.) } \\
& A_{7}\left(\left\{\alpha_{i k}\right\},\left\{\alpha_{j k}\right\},\left\{\beta_{i k}\right\},\left\{\beta_{j k}\right\}\right) \\
& =\frac{1}{N_{7}}\left(\delta_{\beta_{i 1}}^{\alpha_{i 1}} \delta_{\beta_{j 1}}^{\alpha_{j 1}}-\delta_{\beta_{j 1}}^{\alpha_{i 1}} \delta_{\beta_{i 1}}^{\alpha_{j 1}}\right)\left(\delta_{\beta_{i 2}}^{\alpha_{i 2}} \delta_{\beta_{j 2}}^{\alpha_{j 2}}-\delta_{\beta_{j 2}}^{\alpha_{i 2}} \delta_{\beta_{i 2}}^{\alpha_{j 2}}\right) \\
& \left(\delta_{\beta_{i 3}}^{\alpha_{i 3}} \delta_{\beta_{j 3}}^{\alpha_{j 3}}-\delta_{\beta_{j 3}}^{\alpha_{i 3}} \delta_{\beta_{i 3}}^{\alpha_{j 3}}\right)\left(\delta_{\beta_{i 4}}^{\alpha_{i 4}} \delta_{\beta_{j 4}}^{\alpha_{j 4}}-\delta_{\beta_{j 4}}^{\alpha_{i 4}} \delta_{\beta_{i 4}}^{\alpha_{j 4}}\right)
\end{aligned}
$$

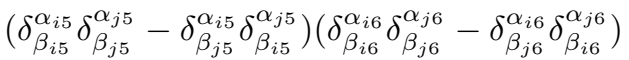

$$
\begin{aligned}
& \left(\delta_{\beta_{i 7}}^{\alpha_{i 7}} \delta_{\beta_{j 7}}^{\alpha_{j 7}}-\delta_{\beta_{j 7}}^{\alpha_{i 7}} \delta_{\beta_{i 7}}^{\alpha_{j 7}}\right)\left(\delta_{\beta_{i 8}}^{\alpha_{i 8}} \delta_{\beta_{i 9}}^{\alpha_{i 9}} \delta_{\beta_{j 8}}^{\alpha_{j 8}} \delta_{\beta_{j 9}}^{\alpha_{j 9}}+\text { sym. }\right) \\
& A_{9}\left(\left\{\alpha_{i k}\right\},\left\{\alpha_{j k}\right\},\left\{\beta_{i k}\right\},\left\{\beta_{j k}\right\}\right) \\
& =\frac{1}{N_{9}}\left(\delta_{\beta_{i 1}}^{\alpha_{i 1}} \delta_{\beta_{j 1}}^{\alpha_{j 1}}-\delta_{\beta_{j 1}}^{\alpha_{i 1}} \delta_{\beta_{i 1}}^{\alpha_{j 1}}\right)\left(\delta_{\beta_{i 2}}^{\alpha_{i 2}} \delta_{\beta_{j 2}}^{\alpha_{j 2}}-\delta_{\beta_{j 2}}^{\alpha_{i 2}} \delta_{\beta_{i 2}}^{\alpha_{j 2}}\right) \\
& \left(\delta_{\beta_{i 3}}^{\alpha_{i 3}} \delta_{\beta_{j 3}}^{\alpha_{j 3}}-\delta_{\beta_{j 3}}^{\alpha_{i 3}} \delta_{\beta_{i 3}}^{\alpha_{j 3}}\right)\left(\delta_{\beta_{i 4}}^{\alpha_{i 4}} \delta_{\beta_{j 4}}^{\alpha_{j 4}}-\delta_{\beta_{j 4}}^{\alpha_{i 4}} \delta_{\beta_{i 4}}^{\alpha_{j 4}}\right) \\
& \left(\delta_{\beta_{i 5}}^{\alpha_{i 5}} \delta_{\beta_{j 5}}^{\alpha_{j 5}}-\delta_{\beta_{j 5}}^{\alpha_{i 5}} \delta_{\beta_{i 5}}^{\alpha_{j 5}}\right)\left(\delta_{\beta_{i 6}}^{\alpha_{i 6}} \delta_{\beta_{j 6}}^{\alpha_{j 6}}-\delta_{\beta_{j 6}}^{\alpha_{i 6}} \delta_{\beta_{i 6}}^{\alpha_{j 6}}\right) \\
& \left(\delta_{\beta_{i 7}}^{\alpha_{i 7}} \delta_{\beta_{j 7}}^{\alpha_{j 7}}-\delta_{\beta_{j 7}}^{\alpha_{i 7}} \delta_{\beta_{i 7}}^{\alpha_{j 7}}\right)\left(\delta_{\beta_{i 8}}^{\alpha_{i 8}} \delta_{\beta_{j 8}}^{\alpha_{j 8}}-\delta_{\beta_{i 8}}^{\alpha_{j 8}} \delta_{\beta_{j 8}}^{\alpha_{i 8}}\right) \\
& \left(\delta_{\beta_{i 9}}^{\alpha_{i 9}} \delta_{\beta_{j 9}}^{\alpha_{j 9}}-\delta_{\beta_{j 9}}^{\alpha_{i 9}} \delta_{\beta_{i 9}}^{\alpha_{j 9}}\right)
\end{aligned}
$$

where sym. means to totally symmetrize the lower indices of the Kronecker delta function $\delta_{\beta}^{\alpha}$, and $N_{q}$ are the nor- malization constants. For any pair $(i j)$. the indices to be set antisymmetric in $A_{q}$ are arbitrary because $\left\{\alpha_{j k}\right\}$ are totally symmetric for each particle $j$. Moreover, from Eq.(8) every $A_{q}$ makes at least 3 and odd number of antisymmetric pairs. Therefore, we find the symmetry that from Eq. (11) $C_{3}$ becomes $-C_{3}$ by exchanging whole group of indices $\left(\alpha_{i 1} \alpha_{i 2} \alpha_{i 3}\right)$ and $\left(\alpha_{j 1} \alpha_{j 2} \alpha_{j 3}\right)$, or by exchanging $\left(\alpha_{i 4} \alpha_{i 5} \alpha_{i 6}\right)$ and $\left(\alpha_{j 4} \alpha_{j 5} \alpha_{j 6}\right)$, or by exchanging $\left(\alpha_{i 7} \alpha_{i 8} \alpha_{i 9}\right)$ and $\left(\alpha_{j 7} \alpha_{j 8} \alpha_{j 9}\right)$. If this symmetry is true for any pair $(i j)$, the argument of the non-degeneracy can be given as the following: first, we fix the indices $\left(\alpha_{i 4} \alpha_{i 5} . . \alpha_{i 9}\right)$ for all particles, namely $i=1, . ., N$. The symmetry that $C_{3}$ picks up a minus sign when exchanging $\left(\alpha_{i 1} \alpha_{i 2} \alpha_{i 3}\right)$ and $\left(\alpha_{j 1} \alpha_{j 2} \alpha_{j 3}\right)$ for any pair $(i j)$ demands the total antisymmetry of the wave function for the first three indices. On the other hand, the dimension spanned by the first three indices is nothing but $\frac{1}{6} \cdot 4 \cdot 5 \cdot 6$, which is equal to the number of particles $N=20$. Then, there is a unique wave function $\Psi$ given by Eq.(4) for the first three indices of every particle, namely $C_{3} \sim \epsilon_{\left(\alpha_{11} \alpha_{12} \alpha_{13}\right)\left(\alpha_{21} \alpha_{22} \alpha_{23}\right) . .\left(\alpha_{N 1} \alpha_{N 2} \alpha_{N 3}\right)}$, where $\epsilon$ is the totally-antisymmetric tensor with respect to exchanging indices of whole $\alpha_{i 1} \alpha_{i 2} \alpha_{i 3}$ and $\alpha_{j 1} \alpha_{j 2} \alpha_{j 3}$. Similarly, applying this argument to the second three and the third three indices of every particle, we obtain $\Psi^{3}$ as the unique ground state wave function for $\chi$.

Now, suppose that there exist another ground state wave function that does not satisfy the symmetry mentioned above for any pair $(i j)$. Let us think of the easiest case. Namely, the symmetry is satisfied for any pair (ij) except for the pair $\left(i^{\prime} j^{\prime}\right)$ that under the exchange of $\left(\alpha_{i^{\prime} 1} \alpha_{i^{\prime} 2} \alpha_{i^{\prime} 3}\right)$ and $\left(\alpha_{j^{\prime} 1} \alpha_{j^{\prime} 2} \alpha_{j^{\prime} 3}\right)$,

$$
C_{3}^{\prime}=\sum\left[-A_{3}+A_{5}-A_{7}-A_{9}\right] C_{3}
$$

where we omit the summation variables. From Eq.(12) and Eq.(10), we can define another variable $C^{\prime \prime}=C_{3}-C_{3}^{\prime}$ for all $(i j)$. It is now clear that the solution given by $C^{\prime \prime}$ does not have the $(8,5,0)$ state for the pair $\left(i^{\prime} j^{\prime}\right)$. However, $C$ " satisfies the symmetry mentioned above. According to the argument above, it has to be $\Psi^{3}$. Then, the contradiction occurs, because one can easily show that $\Psi^{3}$ contains $(8,5,0)$ state for any pair $(i j)$. To reconcile the contradiction, $\Psi^{3}$ has to be the unique solution. This argument can be generalized to more complicate cases or general $p$ cases.

The excitation with fractional charges The natural generalization of Laughlin's quasi-particle/hole operators are given as the following 10 .

$$
\begin{aligned}
& B_{N}^{\dagger}\left(\Phi_{\alpha}\right)=\prod_{i=1}^{N}\left(\Phi_{\alpha} \mathcal{R}_{\alpha \beta} \psi_{\beta}^{i}\right) \\
& B_{N}\left(\Phi_{\alpha}\right)=\prod_{i=1}^{N}\left(\Phi_{\alpha}^{*} \mathcal{R}_{\alpha \beta} \frac{\partial}{\partial \psi_{\beta}^{i}}\right)
\end{aligned}
$$

where $\Phi_{\alpha}$ is a four-component complex spinor with 
$\bar{\Phi}^{\alpha} \Phi_{\alpha}=1$ denoting the position that the excitation is created in $\mathrm{CP}^{3} . \mathcal{R}_{\alpha \beta}$ is the charge conjugate matrix which takes the following form

$$
\mathcal{R}_{\alpha \beta}=\left(\begin{array}{cc}
-i \sigma_{2} & 0 \\
0 & -i \sigma_{2}
\end{array}\right)
$$

$B_{N}^{\dagger}\left(\Phi_{\alpha}\right) \Psi_{N}^{m}\left(B_{N}\left(\Phi_{\alpha}\right) \Psi_{N}^{m}\right)$ describes a hole-like (particlelike) excitation because the size of the system has been enlarged (reduced) by $+(-) \frac{1}{2} m^{2} p^{2}$, where the singleparticle state is described by the $(m p+(-) 1,0,0)$ state. Because $p \sim R^{2}$, these excitations are the fourdimensional objects, namely quasi-4-branes. The extended excitations are not very new to condensed matter physicists. For example, in superfluid, a vortex excitation is a point-like particle in 2 spatial dimensions and a onedimensional string in 3 spatial dimensions. In general, in $D$ spatial dimensions, a vortex is a $(D-2)$-dimensional extended objects. In our case, $\mathrm{CP}^{3}$ is 6 -dimensional. The quantum quasi-4-brane may be regarded as a vortex excitation in the generalized fractional quantum Hall fluid 3, 11,12 .

We can apply Haldane's argument of the fractional charge in our system [10]. In the thermodynamical limit, the number of particle $N \sim \frac{1}{6} p^{3}$, so $p \sim \sqrt[3]{6} N^{\frac{1}{3}}$. Define $p(N, m)=\sqrt[3]{6} m N^{\frac{1}{3}}$ for the fractional case such that the single-particle state is in the $(p(N, m), 0,0)$ state. By changing the field strength, a state with $N_{p}^{\text {ex }}$ particle-like and $N_{h}^{\text {ex }}$ hole-like quasi-4-branes has $p=p(N, m)+\left(N_{h}^{\mathrm{ex}}-N_{p}^{\mathrm{ex}}\right)$. On the other hand, if we fix the field strength and excite the systems by removing (injecting) particles, we have to remove (inject) $\frac{1}{2} m^{2} p^{2} \sim \frac{(\sqrt[3]{6})^{2}}{2} m^{2} N^{\frac{2}{3}}$ particles to make the quasi4-brane. Then, we obtain $p\left(N \pm \frac{(\sqrt[3]{6})^{2}}{2} m^{2} N^{\frac{2}{3}}, m\right)=$ $\sqrt[3]{6} m\left(N \pm \frac{(\sqrt[3]{6})^{2}}{2} m^{2} N^{\frac{2}{3}}\right)^{\frac{1}{3}} \simeq \sqrt[3]{6} m N^{\frac{1}{3}} \pm m^{3}$. In other words, one 4-brane with charge $+(-) 1$ is attached by $m^{3}$ flux. Then, the elementary excitation by changing unit flux carries a fractional charge $q=+(-) 1 / m^{3}$.

Finally, let us discuss about the density correlation function in the fractional case defined by $\rho_{m}\left(x, x^{\prime}\right)=$ $\frac{1}{(N-2) !} \int d x_{3} \cdot d x_{N}\left|\Psi_{N}^{m}\right|^{2}$. For $m=1$ [1], it is given by

$$
\rho_{1}\left(x, x^{\prime}\right)=1-\left|\bar{\psi}_{\alpha}(x) \psi_{\alpha}\left(x^{\prime}\right)\right|^{2 p} .
$$

When one take $x^{\prime}$ to be the north pole of both the orbital and the internal space and let $x$ approach to $x^{\prime}$, then $\rho_{1}\left(x, x^{\prime}\right) \sim 1-e^{-\frac{1}{4 l_{0}^{2}}\left(X_{\mu}^{2}+N_{\alpha}^{2}\right)}$ provided that $R=r$ where $X_{\mu}^{2}=R^{2} \sum_{\mu=1}^{4} x_{\mu}^{2}$ and $N_{\alpha}^{2}=R^{2}\left(n_{1}^{2}+n_{2}^{2}\right)[1]$. We calculate $\rho_{3}\left(x, x^{\prime}\right)$ for $m=3$ and $p=1$ :

$$
\rho_{3}\left(x, x^{\prime}\right)=\left(1-\left|\bar{\psi}_{\alpha}(x) \psi_{\alpha}\left(x^{\prime}\right)\right|^{2}\right)^{3}+O\left(x_{\mu}^{8}, n_{\alpha}^{8}\right)
$$

when $x$ approaches $x^{\prime}$. As two particles are close enough, the higher order vanishes faster than the leading order term. Comparing Eq.(16) and Eq.(17), the density correlation function for general $p$ and $m$ should have the following form

$$
\begin{aligned}
\rho_{m}\left(x, x^{\prime}\right) & \sim\left(1-\left|\bar{\psi}_{\alpha}(x) \psi_{\alpha}\left(x^{\prime}\right)\right|^{2 p}\right)^{m} \\
& \sim\left(1-e^{-\frac{1}{4 l_{0}^{2}}\left(X_{\mu}^{2}+N_{\alpha}^{2}\right)}\right)^{m} .
\end{aligned}
$$

Eq. (18) states that in the filling factor $\nu=1 / \mathrm{m}^{3}$ case the density correlation function vanishes as $m$-th order root when two particles approach to each other. This behavior suggests that the generalized fractional quantum Hall state is an incompressible liquid.

To summarize, similar to the $2 \mathrm{D}$ fractional quantum Hall effect, the 4D FQHS has to be stabilized by a two-body repulsion interaction. We also prove the nondegeneracy of the $4 \mathrm{D}$ FQHS. We found the correspondent elementary excitations of Laughlin's quasi-hole (quasiparticle) in $\mathrm{CP}^{3}$, which can be considered as vortices in $\mathrm{CP}^{3}$. They are extended objects carrying fractional charges $+(-) 1 / m^{3}$. We also discuss the density correlation function in the fractional case, which indicates that the FQHS is an incompressible liquid.

There are several open issues regarding to the FQHS. In Ref. [3], using the effective field-theoretic approach the FQHS can be shown to support 2-dimensional excitations which obey fractional statistics. The correspondent states in our pseudo-potential approach need to be clarified. Also, the edge mode of the FQHS is always an intriguing issue for the future exploration.

We are grateful for the stimulating discussions with Darwin Chang, Dung-Hai Lee, and Naoto Nagaosa. This work is supported by the ERATO-SSS project.

* Electronic address: chern@appi.t.u-tokyo.ac.jp

[1] S.-C. Zhang and J. Hu, Science 294, 823 (2001).

[2] S. Murakami, N. Nagaosa, and S.-C. Zhang, Science 301, 1348 (2003).

[3] B. A. Bernevig, C.-H. Chern, J.-P. Hu, N. Toumbas, and S.-C. Zhang, ANNALS PHYS. 300, 185 (2002).

[4] B. A. Bernevig, J. P. Hu, N. Toumbas, and S. C. Zhang, Physical Review Letters 91, 236803 (2003).

[5] M. Fabinger, JHEP 0205, 037 (2002).

[6] H. Elvang and J. Polchinski, hep-th/0209104 (2002).

[7] J. Hu and S.-C. Zhang, Phys. Rev. B 66, 125301 (2002).

[8] Y. Kimura, Nucl. Phys. B 637, 177 (2002).

[9] R. Laughlin, Phys. Rev. Lett. 50, 1395 (1983).

[10] F. Haldane, Phys. Rev. Lett. 51, 605 (1983).

[11] S.-C. Zhang, T. Hansson, and S. Kivelson, Physical Review Letters 62, 82 (1989).

[12] N. Reed, Physical Review Letters 62, 88 (1989). 